Sialendoscopy in treatment of adult chronic recurrent parotitis without sialolithiasis

Jokela, Johanna

2018-03

Jokela , J , Haapaniemi , A , Mäkitie , A \& Saarinen , R 2018 , ' Sialendoscopy in treatment of adult chronic recurrent parotitis without sialolithiasis ', European Archives of Oto-Rhino-Laryngology , vol. 275 , no. 3 , pp. 775-781 . https://doi.org/10.1007/s00405-017-4854-7

http://hdl.handle.net/10138/300886

https://doi.org/10.1007/s00405-017-4854-7

publishedVersion

Downloaded from Helda, University of Helsinki institutional repository.

This is an electronic reprint of the original article.

This reprint may differ from the original in pagination and typographic detail.

Please cite the original version. 


\title{
Sialendoscopy in treatment of adult chronic recurrent parotitis without sialolithiasis
}

\author{
Johanna Jokela $^{1} \cdot$ Aaro Haapaniemi $^{1} \cdot$ Antti Mäkitie $^{1,2} \cdot$ Riitta Saarinen $^{1}$
}

Received: 25 October 2017 / Accepted: 21 December 2017 / Published online: 30 December 2017

c) Springer-Verlag GmbH Germany, part of Springer Nature 2017

\begin{abstract}
Objective The aim of this prospective study was to evaluate the efficacy of sialendoscopy in the management of adult chronic recurrent parotitis without sialolithiasis. In addition, preliminary results of an initial randomized placebo-controlled trial of single-dose intraductal steroid injection given concurrently with sialendoscopy, are presented.

Methods Forty-nine adult patients with chronic recurrent parotitis without sialoliths were included in this study. They underwent sialendoscopy and were randomized to receive either a concurrent intraductal injection of isotonic saline solution or $125 \mathrm{mg}$ of hydrocortisone. Symptom severity was evaluated with visual analogue scale (VAS) and by recording symptom frequency and course with a multiple-choice questionnaire completed preoperatively and at 3,6, and 12 months after the procedure.

Results The mean VAS score was 5.6 preoperatively and dropped to 2.9 at 3 months, 3.0 at 6 months, and 2.7 at 12 months after the procedure. The VAS score and the frequency of symptoms were significantly lower at $3(p<0.001), 6(p<0.001)$ and $12(p<0.001)$ months after the procedure when compared with the preoperative scores indicating that sialendoscopy reduces the symptoms of recurrent parotitis. However, complete permanent resolution of symptoms was rare. Single-dose steroid injection concomitant to sialendoscopy provided no additional benefit, but the current study is not sufficiently powered to determine a clinical difference between the steroid and non-steroid groups.

Conlusion Sialendoscopy appears to reduce the symptoms of chronic recurrent parotitis. While total permanent symptom remission is rare, sialendoscopy can be considered a safe and relatively efficacious treatment method for this patient group.
\end{abstract}

Keywords Chronic recurrent parotitis $\cdot$ Sialendoscopy $\cdot$ Sialadenitis $\cdot$ Steroids

\section{Introduction}

Chronic recurrent parotitis is an inflammatory condition characterized by periodic or persistent unilateral or bilateral swelling and tenderness of the parotid gland(s). The exact pathogenesis is not fully understood, but the primary pathophysiological event is believed to be due to decreased salivary secretion or obstructive salivary stagnation. Insufficient

Johanna Jokela

johanna.jokela@fimnet.fi

1 Department of Otorhinolaryngology, Head and Neck Surgery, Helsinki University Hospital and University of Helsinki, P.O. Box 263, 00029 HUS Helsinki, Finland

2 Division of Ear, Nose and Throat Diseases, Department of Clinical Sciences, Intervention and Technology, Karolinska Institutet and Karolinska Hospital, Stockholm, Sweden salivary flow can lead to ascending salivary duct infection via the oral cavity and potentially will progress to acute bacterial infection or frequent opportunistic infections [1]. Recurrent inflammation may lead to sialectasis formation, acinar destruction, fibrosis, and chronic inflammatory cell infiltration, thus further reducing salivary flow $[2,3]$. Ductal obstruction may be caused by many etiological factors, including sialoliths, strictures, stenosis, mucous plugs, polyps, and foreign bodies [4]. Non-obstructive factors, such as radiation damage and autoimmune disease, may also decrease saliva secretion and flow [5]. In many cases, however, the etiology is unclear.

Primary treatment of chronic parotitis is conservative, i.e., NSAIDs, sialogogues, mouth rinses, gland massage, and in case of an acute purulent infection, antibiotics $[1,6]$. Invasive approaches include tympanic neurectomy and superficial or total parotidectomy as a final option [1]. Salivary 
duct ligation has also been described, but is currently rarely performed due to high failure rate $[7,8]$.

The development of modern sialendoscopy has allowed improved visual access into the salivary duct system to diagnose and treat obstructive causes. Sialendoscopy can be performed under local anesthesia with minimal morbidity $[9,10]$. Good results have been achieved in the treatment of sialolithiasis and ductal stenosis or strictures [11]. There is some preliminary evidence that sialendoscopy can also relieve symptoms of autoimmune-mediated salivary gland diseases [12]. Despite these advances, patients with chronic recurrent sialadenitis without sialolithiasis often have persistent or recurrent symptoms [13]. Intraductal steroid irrigations are frequently used concurrently with sialendoscopy to manage these patients.

The aim of this study was to prospectively evaluate the efficacy of sialendoscopy in the treatment of chronic recurrent parotitis without sialolithiasis and try to provide preliminary results from the benefits of single-dose intraductal hydrocortisone given concurrently with sialendoscopy.

\section{Materials and methods}

The institutional Research Ethics Committee approved the study design and research permission was granted. The Declaration of Helsinki 2013 guidelines and protocols were followed. Informed consent was obtained from all individual participants included in the study.

This prospective study was conducted at the Department of Otorhinolaryngology-Head and Neck Surgery, Helsinki University Hospital, Helsinki, Finland, between March 2012 and December 2015. In total, 54 patients gave their consent to participate in this study. However, five recruited patients were subsequently excluded due to spontaneous symptom improvement; by the time of the endoscopy these patients no longer fulfilled the inclusion criteria. Overall, 49 patients were included in the study. All patients had a history of recurrent or persistent swelling of the parotid gland(s) with or without pain, which significantly reduced their quality of life. The inclusion criteria were at least three prolonged (over $24 \mathrm{~h}$ ) or ten shorter episodes of parotid swelling during the last 6 months prior to sialendoscopy. Ultrasound examination or, in some cases, MRI was performed before sialendoscopy and patients with sialoliths were excluded from the study.

The sialendoscopies were performed under local anesthesia as day surgery procedures using all-in-one and interventional 1.1 or $1.3 \mathrm{~mm}$ sialendoscopes (Karl Storz: $11573 \mathrm{~A}$ and $11575 \mathrm{~A}$ ). The ductal system was explored under continuous lavage with isotonic saline solution to enhance the visualization and to remove debris and mucus plaques. If strictures were encountered, an attempt was made to gently dilate them with the endoscope itself, a salivary duct probe, or a micro drill. During the endoscopic procedure, the patients were randomized in a double-blind fashion at a 1:1 ratio to receive either $1 \mathrm{~mL}$ of isotonic saline solution (group A, $n=24$ ) or $1 \mathrm{~mL}$ of hydrocortisone $125 \mathrm{mg}$ / $\mathrm{mL}$ (group B, $n=25$ ) intraductally. The $1 \mathrm{~mL}$ of isotonic saline or hydrocortisone was given after the sialendoscopic procedure under visual control, and the gland was emptied from rinsing solution by milking it beforehand. If a patient had bilateral symptoms, sialendoscopy was performed for the gland with more symptoms, and in some cases, for both glands with identical technique. Gland massage was recommended from the following day on. Pre- or postoperative antibiotics were not used.

We designed a questionnaire to assess the severity and frequency of parotid symptoms. The severity of symptoms was evaluated by means of a visual analogue scale (VAS, horizontal line of $10 \mathrm{~cm}$ ) from 0 (no symptoms) to 10 (the worst possible symptoms). Patients were asked to estimate the intensity of symptoms during the last 3 months and mark the average score on the VAS line. The symptom frequency and course were evaluated with categorical questions. Patients were requested to estimate the frequency of symptoms during the last 3 months and mark the most suitable option. These categories appear in Fig. 1. In addition, patients were asked to estimate if their symptoms had resolved completely, decreased significantly, decreased a little, remained unchanged, increased a little or increased significantly during the last 3 months. The patients completed questionnaires and VAS before sialendoscopy and 3, 6, and 12 months after the procedure. An outpatient control visit was conducted on average 3 months after the sialendoscopy.

We gathered data on patient demographics, medical history, dental status (prostheses, implants, bridges etc.,) tobacco smoking, imaging findings, sialendoscopy findings, interventions, complications, and possible further treatments from the medical records and prequestionnaire forms. Stricture was defined as a local narrowing or obstruction of the duct, and duct stenosis as a longer or generalized narrowing of the duct. If the patient had disruptive persistent parotid symptoms at a follow-up visit 3 months after the procedure or later, ductal saline lavage followed by an intraductal cortisone injection could be administered at an outpatient clinic. These patients were excluded from later analyses. Patients with incomplete follow-up information at some time point were excluded from analyzes at that point. Eventually, 49 patients were included for the analyses at 3 months postoperatively, 38 patients at 6 months postoperatively and 42 patients at 12 months postoperatively.

Descriptive statistics were used to analyze the demographic and clinical characteristics of the study population. The answers are given as absolute numbers and percentages or mean values. 


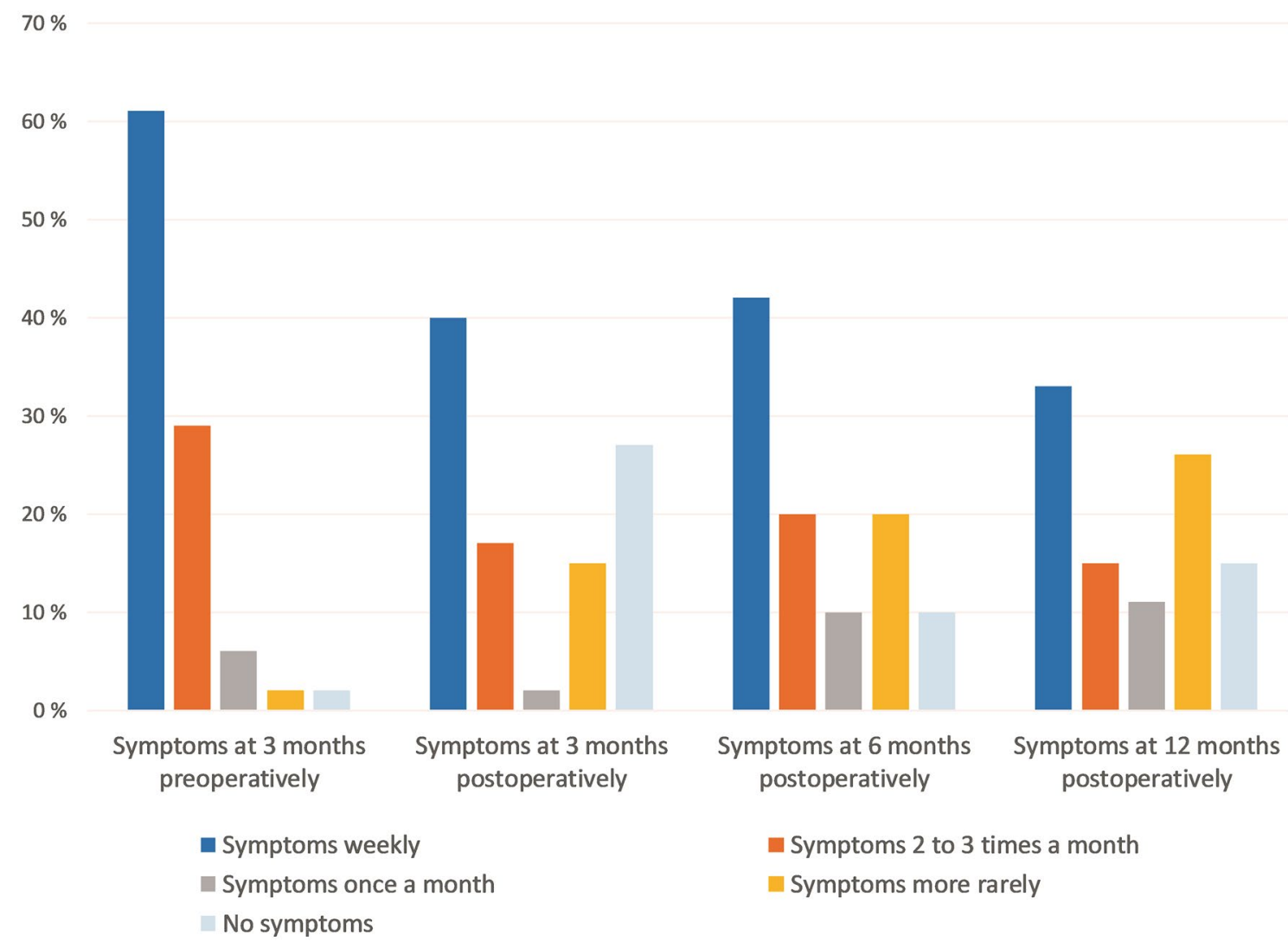

Fig. 1 Frequency of symptoms at 3 months preoperatively and at 3, 6, and 12 months postoperatively

Comparison of symptom frequency and VAS scores before and after sialendoscopy were performed using the Wilcoxon non-parametric signed-rank test by analyzing the within-subject change. The comparability of subgroups A and B were assessed using Chi-square test and Fisher's exact tests. The frequency of symptoms and VAS scores were compared between groups $\mathrm{A}$ and $\mathrm{B}$ using equal-variance $t$ test and analysis of covariance report.

Relationships between demographic, clinical and endoscopic variables, and VAS scores were assessed by means of the $t$ test, the analysis of covariance report and the Spearman rank-correlation test.

A $p$ value less than 0.05 was considered to indicate statistical significance. The analyses were performed using NCSS 8 statistical software.

\section{Results}

A total of 49 patients participated in this study. Demographic and clinical characteristics of the whole series are presented in Table 1. The mean patient age was 53 years (range 21-75). Thirty-three (67\%) patients were women, 6 $(12 \%)$ had been diagnosed with an autoimmune disease and
$31(63 \%)$ were smokers or had stopped smoking recently. All patients had recurrent swelling of the parotid gland with tenderness of the gland, 19 (39\%) had also significant pain, and $8(16 \%)$ had suffered from recurrent bacterial infections. The average symptom duration was 7.2 years (median 4.5 years; range $0.5-41)$. Twelve (24\%) patients had had their first symptoms decades ago, after which they had been symptom-free until the symptoms recurred a few years prior to the sialendoscopy.

Ultrasound was performed for 47 (96\%) patients preoperatively and MRI for $3(6 \%)$. Ductal dilation $(n=11,22 \%)$ and heterogeneity of the parenchyma with small hypoechoic or anechoic areas $(n=10,20 \%)$ were the most common imaging findings. Small cysts were found in two MRIs.

The most frequent finding in sialendoscopy were mucous plugs $(n=28,57 \%)$, followed by stricture of the duct $(n=21,43 \%)$, dilation of the duct $(n=10,20 \%)$, stenosis of the duct $(n=8,16 \%)$, and sialodochitis $(n=5,10 \%)$. In $12(24 \%)$ patients only a small amount of mucous exudate was found in an otherwise normal ductal system. A stricture was opened and dilated in 17 (35\%) patients. Eight patients (16\%) had bilateral sialendoscopy.

Forty-seven (96\%) patients had a follow-up visit approximately 3 months after the operation. Further visits were 
Table 1 Demographic, clinical, and endoscopic characteristics

\begin{tabular}{|c|c|}
\hline Characteristics & Total (49 patients) \\
\hline \multicolumn{2}{|l|}{ Demographic and preoperative characteristics } \\
\hline Women $(\%)$ & $33(67)$ \\
\hline Mean age (years) & 53 \\
\hline Tobacco smoking (\%) & $31(63)$ \\
\hline Autoimmune disease (\%) & $6(12)$ \\
\hline Duration of symptoms (years) & 7.2 \\
\hline \multicolumn{2}{|l|}{ Symptoms (\%) } \\
\hline Swelling & $49(100)$ \\
\hline Pain & $19(39)$ \\
\hline Recurrent bacterial infections & $8(16)$ \\
\hline \multicolumn{2}{|l|}{ Size $(\%)$} \\
\hline Right & $9(18)$ \\
\hline Left & $19(39)$ \\
\hline Bilateral & $21(43)$ \\
\hline Symptoms also in submandibular gland(s) (\%) & $4(8)$ \\
\hline VAS score & 5.6 \\
\hline \multicolumn{2}{|l|}{ Endoscopic characteristics } \\
\hline \multicolumn{2}{|l|}{ Endoscopic findings (\%) } \\
\hline Mucous plugs & $28(57)$ \\
\hline Stricture & $21(43)$ \\
\hline Stenosis & $8(16)$ \\
\hline Dilation & $10(20)$ \\
\hline Sialodochitis & $5(10)$ \\
\hline Normal & $12(24)$ \\
\hline Stricture dilation (\%) & $17(35)$ \\
\hline \multicolumn{2}{|l|}{ Size $(\%)$} \\
\hline Right & $15(31)$ \\
\hline Left & $26(53)$ \\
\hline Bilateral & $8(16)$ \\
\hline \multicolumn{2}{|l|}{ Postoperative characteristics } \\
\hline \multicolumn{2}{|l|}{ Complications } \\
\hline Bacterial infection & $1(2)$ \\
\hline \multicolumn{2}{|l|}{ VAS score } \\
\hline 3 months postoperatively & 2.9 \\
\hline 6 months postoperatively & 3.0 \\
\hline 12 months postoperatively & 2.7 \\
\hline \multicolumn{2}{|l|}{ Other treatments $(\%)$} \\
\hline Steroid irrigation & $6(12)$ \\
\hline Botox injection & $1(2)$ \\
\hline Tympanic neurectomy & $1(2)$ \\
\hline
\end{tabular}

arranged as needed. Six (12\%) patients received intraductal cortisone injection(s) 3-8 months after the sialendoscopy at an outpatient clinic. One of these patients also received botulinum toxin A injections, and another patient underwent tympanic neurectomy. These patients were excluded from future analyses.

The mean VAS score was 5.6 preoperatively and 2.9 at 3 months, 3.0 at 6 months, and 2.7 at 12 months postoperatively in the whole study group. The average VAS score change was $-2.7,-2.6$ and -2.9 at 3,6 and 12 months postoperatively, respectively. The VAS score was significantly lower at $3(p<0.001), 6(p<0.001)$, and 12 $(p<0.001)$ months after the operation compared to the preoperative VAS score when the change was measured individually. The symptom frequency was also significantly lower when comparing the symptoms at 3 months preoperatively to the symptoms at 3 months postoperatively $(p<0.001)$, at 6 months postoperatively $(p<0.001)$, and at 12 months postoperatively $(p<0.001)$. Patients with higher VAS scores preoperatively had a smaller VAS change postoperatively $(p=0.024)$.

Gender, age, clinical characteristics (autoimmune disease, tobacco smoking, teeth status), duration of symptoms before sialendoscopy, endoscopic findings (mucous plugs, stricture, stenosis, dilation of the duct, sialodochitis), or dilation of a stricture did not correlate with a better or worse postoperative result when measured by means of VAS score change.

The symptom frequency at 3 months preoperatively and at 3,6 , and 12 months postoperatively is shown in Fig. 1 . At 3 months after the operation, $27 \%$ of the patients were symptom-free, symptoms decreased significantly in $31 \%$, decreased slightly in $20 \%$, remained unchanged in $18 \%$, and increased slightly in $4 \%$ based on the patients' selfassessment. However, at 12 months postoperatively only one patient (2\%) had been symptom-free for the whole follow-up time.

In subgroup analyses, no significant difference was observed between the saline group (group A) and the hydrocortisone group (group B) in VAS scores or in symptom frequency at 3 or 12 months postoperatively. The mean and median VAS scores of groups A and B at each time point are presented in Fig. 2. Groups A and B were comparable in the terms of demographic characteristics (gender, age), clinical characteristics (autoimmune disease, tobacco smoking, teeth status), endoscopic findings, and preoperative VAS scores, as well as the duration and frequency of symptoms before sialendoscopy.

\section{Discussion}

The management of chronic parotitis remains challenging and primary treatment consists of conservative approaches. The aim of treatment is to stimulate saliva secretion and to maintain good oral hygiene to reduce the risk of ascending infections. These methods have the potential to control the symptoms in mild cases [8]. However, there are only few studies published on conservative treatment, [1] and the number of studies on the treatment of chronic recurrent parotitis with sialendoscopy is also limited $[11,14,15]$. Therefore, we performed a prospective study on 49 adult 


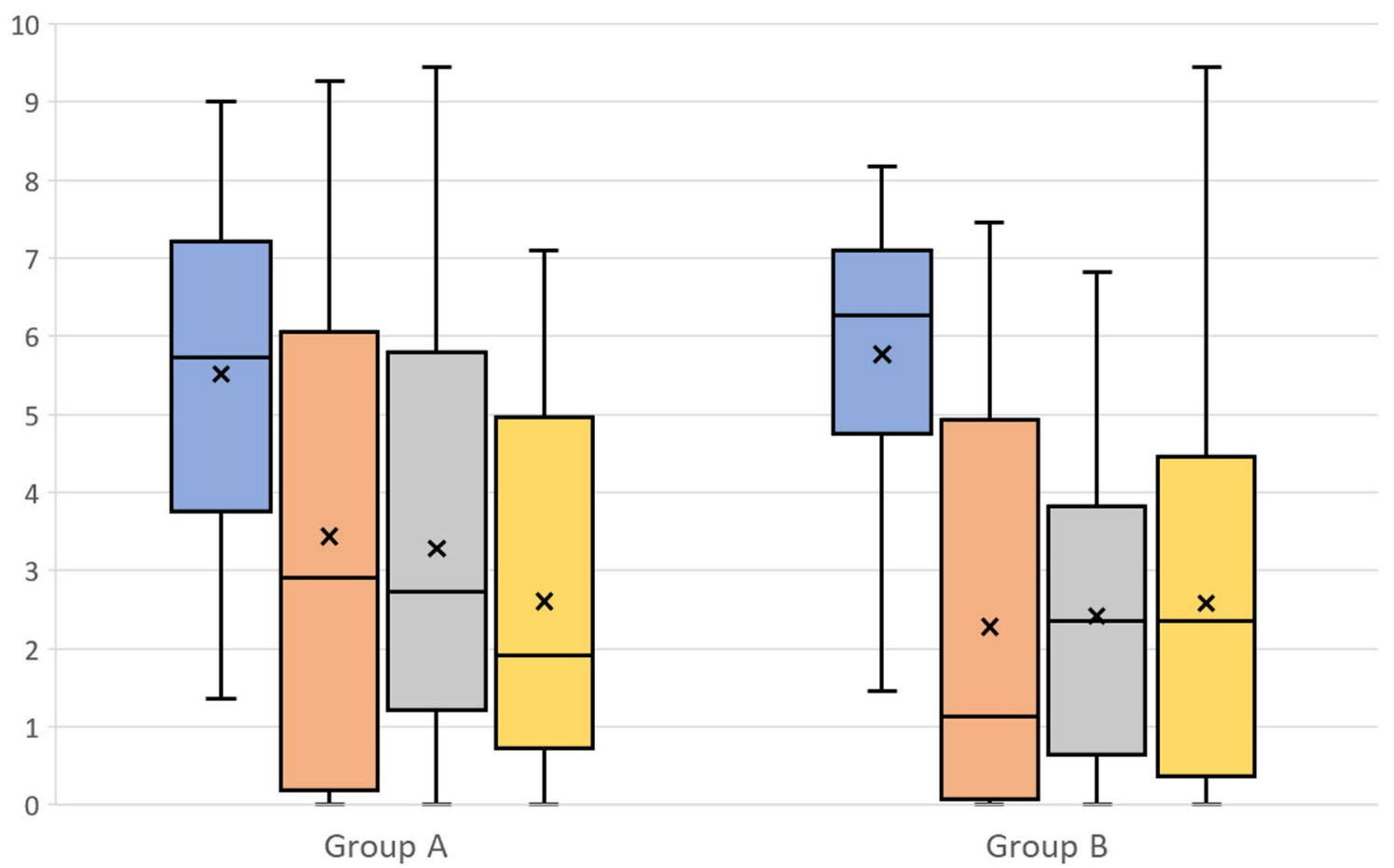

\section{VAS at 3 months preoperatively \\ VAS at 6 months postoperatively \\ VAS at 3 months postoperatively \\ VAS at 12 months postoperatively}

Fig. 2 The VAS scores of groups A and B at 3 months preoperatively and at 3, 6, and 12 months postoperatively. The whiskers represent the highest and the lowest VAS scores, the horizontal line represents the median VAS score and the cross represents the mean VAS score

patients with chronic recurrent parotitis to evaluate the efficacy of sialendoscopy with or wihtout a concurrent singledose intraductal corticosteroid.

A retrospective study by Watkin et al. concluded that out of 30 adults with chronic parotitis with punctuate sialectasis but with no sialoliths on sialography, $56 \%$ showed spontaneous improvement with symptomatic treatment alone [16]. In our study, symptoms were substantially reduced in five patients while waiting for endoscopy and their procedure was accordingly cancelled. It is clear, that a wait-and-see approach may be considered for patients with mild symptoms, since it seems that the disease has a fluctuating natural course and some symptoms tend to resolve spontaneously.

It has been proposed that serum proteins accumulate in the salivary ducts and further coagulate as a consequence of gland inflammation, thus causing obstruction, further inflammation, and damage. Hence, Baurmash suggests that treatment should be directed against both gland inflammation with steroids as well as precipitated serum proteins with rapid and complete removal via dilation, saline irrigation, and milking of the gland [7]. Intraductal instillation of different agents, such as tetracycline or methyl violet (which theoretically induce acinar atrophy), has also been proposed for treatment of chronic parotitis [8, 17]. Surgical management approaches include salivary duct ligation, tympanic neurectomy, and parotidectomy. Ductoplasty outcomes have been disappointing with a high failure rate [1]. In a series of 49 patients, favorable results were achieved by tympanic neurectomy, with resolution or reduction of symptoms in $82 \%$ of cases [18]. Success rates of 90 to $100 \%$ have been reported after superficial or total parotidectomy [1, 19, 20]. In the present study, tympanic neurectomy was performed on one patient with subsequent symptom relief. One patient received botulinum toxin $\mathrm{A}$, which temporarily alleviated her symptoms. During the last decade in our department, we have not included parotidectomy in the management of patients with chronic recurrent parotitis due to its invasive nature, risk of complications, and due to the success achieved with more conservative approaches.

The development of sialendoscopy has improved both the diagnosis and treatment of obstructive sialadenitis [4, 9]. It seems that also patients with inflammatory conditions and autoimmune disease may benefit from sialendoscopy $[12,21,22]$. Based on our results, sialendoscopy reduces the symptoms of chronic recurrent parotitis. Sialendoscopic findings, demographic or clinical characteristics had no 
correlation with clinical outcome, but it seems that patients with higher VAS scores preoperatively had smaller reduction of symptoms. Over three quarters of the patients experienced subjective symptom improvement 3 months postoperatively. Still, over $20 \%$ of patients did not benefit from sialendoscopy and during a 12-month follow up, only one patient remained completely asymptomatic. This might be due to irreversible changes in the gland caused by chronic sialadenitis and decreased salivary flow, which predispose to new obstructive changes and inflammation. The role of repeated procedures in this patient group should be further studied.

Simple lavage of the ductal system can also help remove debris and dilate the duct with hydrostatic pressure and, therefore, the benefit of the endoscopy itself remains somewhat unclear. The advantage of sialendoscopy is the possibility to simultaneously treat obstructive pathology, such as occult sialoliths or strictures. Sialendoscopy also enables more effective irrigation under visual control.

Intraductal steroid concurrently with sialendoscopy is widely used especially in cases of chronic inflammation, stricture dilation, and stenosis $[11,23]$. However, there are only a limited number of studies investigating the role of intraductal steroids in treatment of chronic sialadenitis. In a randomized cross-sectional study on 54 patients, Capaccio et al. concluded that a combination of interventional sialendoscopy followed by repeated ductal steroid irrigations at the outpatient clinic produced a better outcome than sialendoscopy alone in the treatment of recurrent sine causa sialadenitis [24]. Same kind of preliminary results were found in a study of 22 patients with Sjögren syndrome and major salivary gland swelling [25]. Intraductal steroids were also evaluated and found efficacious in pediatric patients with juvenile recurrent parotitis treated with ductal hydrocortisone infusion through a catheter inserted in the parotid duct, [26] and in a study on salivary glands affected by autoimmune disease treated with sialendoscopy and an intraductal hydrocortisone injection [12]. However, there were no control groups in these studies. Delagnes et al. observed that the use of triamcinolone irrigation concurrently with sialendoscopy was associated with better symptom resolution in patients with sialadenitis without sialoliths. However, in multivariate analysis, this finding was not statistically significant [22]. We found no additional benefit in symptom relief from a single-dose intraductal steroid.

There is some heterogeneity in our study population. We had a female predominance, which has also been detected in other studies of chronic parotitis [5]. Six patients had been diagnosed with autoimmune disease and the duration of symptoms varied from 6 months to decades. Patients with longer symptom duration $(p=0.04)$ and female patients $(p=0.045)$ had higher VAS scores preoperatively, but no correlation with postoperative results was found. Interestingly, $63 \%$ of patients were smokers or had stopped smoking recently. According to the Finnish National Institute for Health and Welfare, less than $20 \%$ of Finnish adults currently smoke. The etiological role of smoking in chronic parotitis remains unclear, but this poses the question if tobacco can predispose to chronic sialadenitis. In our series, the follow-up period of 12 months was longer than that presented in many previous studies, but still relatively short for the observation of this patient group with chronic recurrent symptoms.

Our study has some limitations. The lack of a validated symptom outcome questionnaire may be considered a substantial weakness. In our study setting, the patients had to assess the average frequency and intensity of their symptoms during last 3 months and this may have led to some memory bias. In addition, our study cohort was relatively small particularly for subgroup analysis. Postoperative VAS scores were highly variable and with this group size only a substantial effect of steroids would have been detectable. It is possible that intraductal steroids have some benefits, but we were not able to demonstrate them. Hence, further studies with larger patient series are needed to clarify the role of intraductal steroid therapy in the treatment of chronic recurrent parotitis.

\section{Conclusion}

Sialendoscopy appears to reduce the symptoms of adult chronic recurrent parotitis without sialolithiasis, and can be considered a safe and efficacious treatment method. However, complete permanent resolution of symptoms is rare. We did not find any significant benefit from a singledose intraductal steroid injection concomitant with sialendoscopy but the present series is not sufficiently powered to determine a clinical difference between the steroid and non-steroid groups.

Funding This study was funded by the Helsinki University Hospital Research Fund.

\section{Compliance with ethical standards}

Conflict of interest The authors declare that they have no conflict of interest.

Ethical approval All procedures performed in studies involving human participants were in accordance with the ethical standards of the institutional and/or national research committee and with the 1964 Helsinki declaration and its later amendments or comparable ethical standards.

Informed consent Informed consent was obtained from all individual participants included in the study. 


\section{References}

1. Motamed M, Laugharne D, Bradley PJ (2003) Management of chronic parotitis: a review. J Laryngol Otol 117:521-526. https:// doi.org/10.1258/002221503322112923

2. Wang SL, Zou ZJ, Wu QG, Sun KH (1992) Sialographic changes related to clinical and pathologic findings in chronic obstructive parotitis. Int J Oral Maxillofac Surg 21:364-368

3. Bhatty MA, Piggot TA, Soames JV, McLean NR (1998) Chronic non-specific parotid sialadenitis. Br J Plast Surg 51:517-521

4. Nahlieli O, Baruchin AM (2000) Long-term experience with endoscopic diagnosis and treatment of salivary gland inflammatory diseases. Laryngoscope 110:988-993

5. Seifert G (1997) Aetiological and histological classification of sialadenitis. Pathologica 89:7-17

6. Nahlieli O, Bar T, Shacham R, Eliav E, Hecht-Nakar L (2004) Management of Chronic Recurrent Parotitis: Current Therapy. J Oral Maxillofac Surg 62:1150-1155

7. Baurmash HD (2004) Chronic recurrent parotitis: a closer look at its origin, diagnosis, and management. J Oral Maxillofac Surg 62:1010-1018

8. Wang S, Marchal F, Zou Z, Zhou J, Qi S (2009) Classification and management of chronic sialadenitis of the parotid gland. J Oral Rehabil 36:2-8. https://doi. org/10.1111/j.1365-2842.2008.01896.x

9. Marchal F, Dulguerov P, Becker M, Barki G, Disant F, Lehmann W (2001) Specificity of parotid sialendoscopy. Laryngoscope 111:264-271. https://doi. org/10.1097/00005537-200102000-00015

10. Jokela J, Haapaniemi A, Makitie A, Saarinen R (2017) Sialendoscopy under local anaesthesia. Acta Otolaryngol 137:310-314. https://doi.org/10.1080/00016489.2016.1230681

11. Koch M, Iro H, Klintworth N, Psychogios G, Zenk J (2012) Results of minimally invasive gland-preserving treatment in different types of parotid duct stenosis. Arch Otolaryngol Head Neck Surg 138:804-810. https://doi.org/10.1001/archoto.2012.1618

12. Shacham R, Puterman MB, Ohana N, Nahlieli O (2011) Endoscopic treatment of salivary glands affected by autoimmune diseases. J Oral Maxillofac Surg 69:476-481. https://doi. org/10.1016/j.joms.2010.10.002

13. Jokela J, Haapaniemi A, Ojala J, Makitie A, Saarinen R (2016) Sialendoscopy in sialadenitis: an unselected cohort of 228 patients. Clin Otolaryngol 41:416-420. https://doi.org/10.1111/ coa. 12531

14. Wu CB, Xue L, Zhang B, Sun NN, Zhou Q (2015) Sialendoscopyassisted treatment for chronic obstructive parotitis-our treatment strategy with 31 patients. J Oral Maxillofac Surg 73:1524-1531. https://doi.org/10.1016/j.joms.2015.02.024

15. Heineman TE, Kacker A, Kutler DI (2015) Idiopathic chronic parotitis: imaging findings and sialendoscopic response. ORL J Otorhinolaryngol Relat Spec 77:302-309. https://doi. org/10.1159/000438760

16. Watkin GT, Hobsley M (1986) Natural history of patients with recurrent parotitis and punctate sialectasis. Br J Surg 73:745-748

17. Bowling DM, Ferry G, Rauch SD, Goodman ML (1994) Intraductal tetracycline therapy for the treatment of chronic recurrent parotitis. Ear Nose Throat J 73:262-274

18. Vasama JP (2000) Tympanic neurectomy and chronic parotitis. Acta Otolaryngol 120:995-998

19. Sharma R (2013) Superficial parotidectomy for chronic parotid sialadenitis. Int J Oral Maxillofac Surg 42:129-132. https://doi. org/10.1016/j.ijom.2012.04.003

20. Nouraei SA, Ismail Y, McLean NR, Thomson PJ, Milner RH, Welch AR (2007) Surgical treatment of chronic parotid sialadenitis. J Laryngol Otol 121:880-884

21. Guo YF, Sun NN, Wu CB, Xue L, Zhou Q (2017) Sialendoscopy-assisted treatment for chronic obstructive parotitis related to Sjogren syndrome. Oral Surg Oral Med Oral Pathol Oral Radiol 123:305-309

22. Delagnes EA, Aubin-Pouliot A, Zheng M, Chang JL, Ryan WR (2016) Sialadenitis without sialolithiasis: prospective outcomes after sialendoscopy-assisted salivary duct surgery. Laryngoscope 127:1073-1079. https://doi.org/10.1002/lary.26308

23. Gillespie MB, Intaphan J, Nguyen SA (2011) Endoscopic-assisted management of chronic sialadenitis. Head Neck 33:1346-1351. https://doi.org/10.1002/hed.21620

24. Capaccio P, Torretta S, Di Pasquale D, Rossi V, Pignataro L (2017) The role of interventional sialendoscopy and intraductal steroid therapy in patients with recurrent sine causa sialadenitis: a prospective cross-sectional study. Clin Otolaryngol 42:148-155. https://doi.org/10.1111/coa.12681

25. Capaccio P, Canzi P, Torretta $S$ et al (2017) Combined interventional sialendoscopy and intraductal steroid therapy for recurrent sialadenitis in Sjogren's syndrome: results of a pilot monocentric trial. Clin Otolaryngol. https://doi.org/10.1111/coa.12911

26. Roby BB, Mattingly J, Jensen EL, Gao D, Chan KH (2015) Treatment of juvenile recurrent parotitis of childhood: an analysis of effectiveness. JAMA Otolaryngol Head Neck Surg 141:126-129. https://doi.org/10.1001/jamaoto.2014.3036 